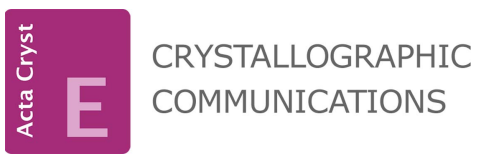

ISSN 2056-9890

Received 19 March 2015

Accepted 22 May 2015

Edited by A. J. Lough, University of Toronto, Canada

Keywords: crystal structure; diphosphine disulfide; copper; $\mathrm{C}-\mathrm{H}$. . F hydrogen bonding

CCDC reference: 1402327

Supporting information: this article has supporting information at journals.iucr.org/e

\section{Crystal structure of bis(1,1,2,2-tetramethyl- diphosphane-1,2-dithione- $\left.\kappa^{2} S, S^{\prime}\right) \operatorname{copper}(\mathrm{I})$ tetrafluoridoborate}

\author{
Peter W. R. Corfield ${ }^{\mathrm{a} *}$ and Uwe Seeler ${ }^{\mathrm{b}}$
}

${ }^{\mathbf{a}}$ Department of Chemistry, Fordham University, 441 East Fordham Road, Bronx, NY 10458, USA, and ${ }^{\mathbf{b}}$ Department of Chemistry, The Ohio State University, Columbus, Ohio 43210, USA. *Correspondence e-mail: pcorfield@fordham.edu

In the title compound, $\left[\mathrm{Cu}\left(\mathrm{C}_{4} \mathrm{H}_{12} \mathrm{P}_{2} \mathrm{~S}_{2}\right)_{2}\right] \mathrm{BF}_{4}$, both diphosphine disulfide molecules bind to the $\mathrm{Cu}^{\mathrm{I}}$ atom, as chelating ligands via the $\mathrm{S}$ atoms, forming a monovalent cation with a slightly distorted tetrahedral coordination around the $\mathrm{Cu}^{\mathrm{I}}$ atom. The average $\mathrm{Cu}-\mathrm{S}$ distance is 2.350 (15) $\AA$, with small but possibly significant differences within each chelate ring. Ligand $\mathrm{P}=\mathrm{S}$ distances average 1.964 (3) $\AA$, and the P-P distances are 2.2262 (13) and 2.2166 (14) $\AA$. The ligand chelate rings are twisted in opposite directions, with one in the $\lambda$ and one in the $\delta$ configuration. Although the anisotropic displacement parameters of the $\mathrm{F}$ atoms of the anion are quite large compared to that of the B atom, difference Fourier syntheses indicate only one set of sites for the F atoms. In the crystal, possible $\mathrm{C}-\mathrm{H} \cdots \mathrm{F}$ hydrogen bonds may stabilize the orientation. The $\mathrm{B}-\mathrm{F}$ distances, uncorrected for libration, average 1.359 (6) $\AA$.

\section{Chemical context}

The title compound was one of a number of phosphine sulfide copper complexes synthesized by Devon Meek and his group (Meek \& Nicpon, 1965). Early reports by Meek and coworkers and by Cotton et al. (1974a) on coordination complexes of diphosphinedisulfide ligands indicated the chelating mode for these ligands to metals such as $\mathrm{Cu}^{\mathrm{I}}$ as only one of several bonding possibilities, particularly as the chelating model involves rotation about the $\mathrm{P}-\mathrm{P}$ bond from the trans conformation found in the structure of the free ligands (see, for example, Lee \& Goodacre, 1971). Indeed, the tetramethyldiphosphinedisulfide ligand was shown in one case to bridge copper atoms forming a polymeric chain (Cotton et $a l ., 1974 b)$. Our work was initiated to verify the chelating structure that had been predicted for the present compound.<smiles></smiles>

$\mathrm{BF}_{4}^{-}$

We have reported this structure previously at the 1973 winter meeting of The American Crystallographic Association. The crystal structure of the corresponding hexafluoridophosphate salt has been reported by Liu et al. (2003). 


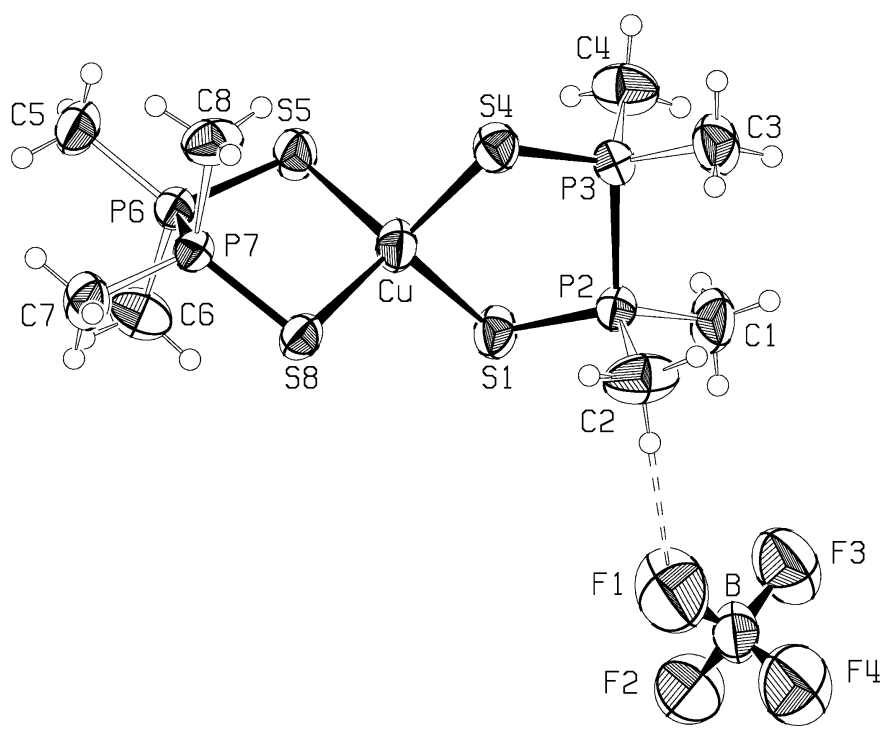

Figure 1

The molecular structure of the title compound, with displacement ellipsoids at the $50 \%$ level. The dashed line indicates a hydrogen bond.

\section{Structural commentary}

In this reported structure, both diphosphine disulfide molecules bind to the $\mathrm{Cu}^{\mathrm{I}}$ atom as chelating ligands via the $\mathrm{S}$ atoms, forming a monovalent cation with a slightly distorted tetrahedral coordination around the $\mathrm{Cu}^{\mathrm{I}}$ (Fig. 1). Liu et al. (2003) have described the structure of the $\mathrm{PF}_{6}{ }^{-}$salt of the present cation, as well as that of the corresponding silver salt.

Selected bond lengths and angles are given in Table 1. The average $\mathrm{Cu}-\mathrm{S}$ distance is 2.350 (15) $\AA$, and distances vary by up to $0.065 \AA$. The chelate $\mathrm{S}-\mathrm{Cu}-\mathrm{S}$ angles are 105.69 (3) and $106.94(5)^{\circ}$, smaller than the other $\mathrm{S}-\mathrm{Cu}-\mathrm{S}$ angles, which vary from $109.10(3)$ to $114.02(4)^{\circ}$ and average $111.1(10)^{\circ}$. Ligand $\mathrm{P}=\mathrm{S}$ distances are more constant, with an average of 1.964 (3) $\AA$, and the $\mathrm{P}-\mathrm{P}$ distances are 2.2262 (13) and 2.2166 (14) $\AA$. The ligand chelate rings are twisted in the $\lambda$ and $\delta$ configurations for $\mathrm{S}_{1} \mathrm{P}_{2} \mathrm{P}_{3} \mathrm{~S}_{4}$ and $\mathrm{S}_{5} \mathrm{P}_{6} \mathrm{P}_{7} \mathrm{~S}_{8}$, respectively, with torsional angles about the $\mathrm{P}-\mathrm{P}$ bonds of 47.97 (6) and $-56.37(6)^{\circ}$. The geometry of the cation, including the slight distortions from regular tetrahedral geometry at the $\mathrm{Cu}^{\mathrm{I}}$ atom, is very similar to that seen by Liu et al. (2003).

The $\mathrm{BF}_{4}{ }^{-}$anion has regular tetrahedral geometry, with an average $\mathrm{F}-\mathrm{B}-\mathrm{F}$ angle of $109.5(6)^{\circ}$ and an average $\mathrm{B}-\mathrm{F}$ distance of 1.359 (6) $\AA$, with distances ranging from 1.347 (5) to $1.370(5) \AA$.

\section{Supramolecular features}

The packing arrangement in the unit cell is shown in Fig. 2. There are no unusual features. The shortest intermolecular contacts not involving $\mathrm{F}$ atoms are $\mathrm{H} 4 A-\mathrm{H} 8 A\left(x, \frac{1}{2}-y, \frac{1}{2}+z\right)$, at $2.42 \AA$ and $\mathrm{H} 7 B-\mathrm{H} 7 C(-x,-y,-1-z)$, at $2.68 \AA$.

A number of recent structural papers in this journal have postulated that $\mathrm{C}-\mathrm{H}$. . O hydrogen bonds were contributing to packing of organic structures (see, for example: Salas et al., 2011; Corfield et al., 2014). This led us to investigate the
Table 1

Selected geometric parameters $\left(\AA{ }^{\circ}\right)$.

\begin{tabular}{lclr}
\hline $\mathrm{Cu}-\mathrm{S} 1$ & $2.3133(15)$ & $\mathrm{Cu}-\mathrm{S} 4$ & $2.3780(17)$ \\
$\mathrm{Cu}-\mathrm{S} 5$ & $2.3719(14)$ & $\mathrm{Cu}-\mathrm{S} 8$ & $2.3383(13)$ \\
$\mathrm{S} 1-\mathrm{Cu}-\mathrm{S} 4$ & $105.69(3)$ & $\mathrm{S} 1-\mathrm{Cu}-\mathrm{S} 5$ & $109.10(3)$ \\
$\mathrm{S} 5-\mathrm{Cu}-\mathrm{S} 8$ & $106.94(5)$ & $\mathrm{S} 4-\mathrm{Cu}-\mathrm{S} 5$ & $110.67(4)$ \\
$\mathrm{S} 1-\mathrm{Cu}-\mathrm{S} 8$ & $114.02(4)$ & $\mathrm{S} 4-\mathrm{Cu}-\mathrm{S} 8$ & $110.46(4)$ \\
\hline
\end{tabular}

Table 2

Hydrogen-bond geometry $\left(\AA{ }^{\circ}\right)$.

\begin{tabular}{lllll}
\hline$A \cdots \mathrm{H}-D$ & $A \cdots \mathrm{H}$ & $\mathrm{H}-D$ & $A \cdots D$ & $A \cdots \mathrm{H}-D$ \\
\hline $\mathrm{F} 1 \cdots \mathrm{H} 2 C-\mathrm{C} 2$ & 2.46 & 0.96 & $3.397(5)$ & 166.6 \\
$\mathrm{~F} 1 \cdots \mathrm{H} 5 B-\mathrm{C} 5^{\mathrm{i}}$ & 2.57 & 0.96 & $3.465(5)$ & 155.8 \\
$\mathrm{~F} 2 \cdots \mathrm{H} 7 B-\mathrm{C} 7^{\mathrm{i}}$ & 2.52 & 0.96 & $3.453(4)$ & 163.7 \\
$\mathrm{~F} 3 \cdots \mathrm{H} 1 C-\mathrm{C} 1^{\mathrm{ii}}$ & 2.45 & 0.96 & $3.378(5)$ & 163.5 \\
$\mathrm{~F} 3 \cdots \mathrm{H} 8 B-\mathrm{C} 8^{\mathrm{iii}}$ & 2.50 & 0.96 & $3.454(5)$ & 170.6 \\
$\mathrm{~F} 4 \cdots \mathrm{H} 1 C-\mathrm{C} 1^{\mathrm{ii}}$ & 2.60 & 0.96 & $3.430(5)$ & 144.6 \\
\hline
\end{tabular}

Symmetry codes: (i) $-x,-y+1,-z$; (ii) $-x+1,-y+1,-z+1$; (iii) $x, y, z+1$.

possibility that $\mathrm{F} \cdots \mathrm{H}-\mathrm{C}$ hydrogen bonds were stabilizing the orientation of the $\mathrm{BF}_{4}{ }^{-}$ion. We list six putative $\mathrm{F} \cdots \mathrm{H}-\mathrm{C}$ hydrogen bonds in Table 2, and they are represented in Fig. 2. F...C distances are all less than $3.5 \AA$, and F. . H distances range from 2.45 to $2.60 \AA$, while angles at the $\mathrm{H}$ atoms are reasonably close to linear.

\section{Database survey}

A search of the in the Cambridge Structure Database (CSD, Version 5.35; Groom \& Allen, 2014) with a substructure

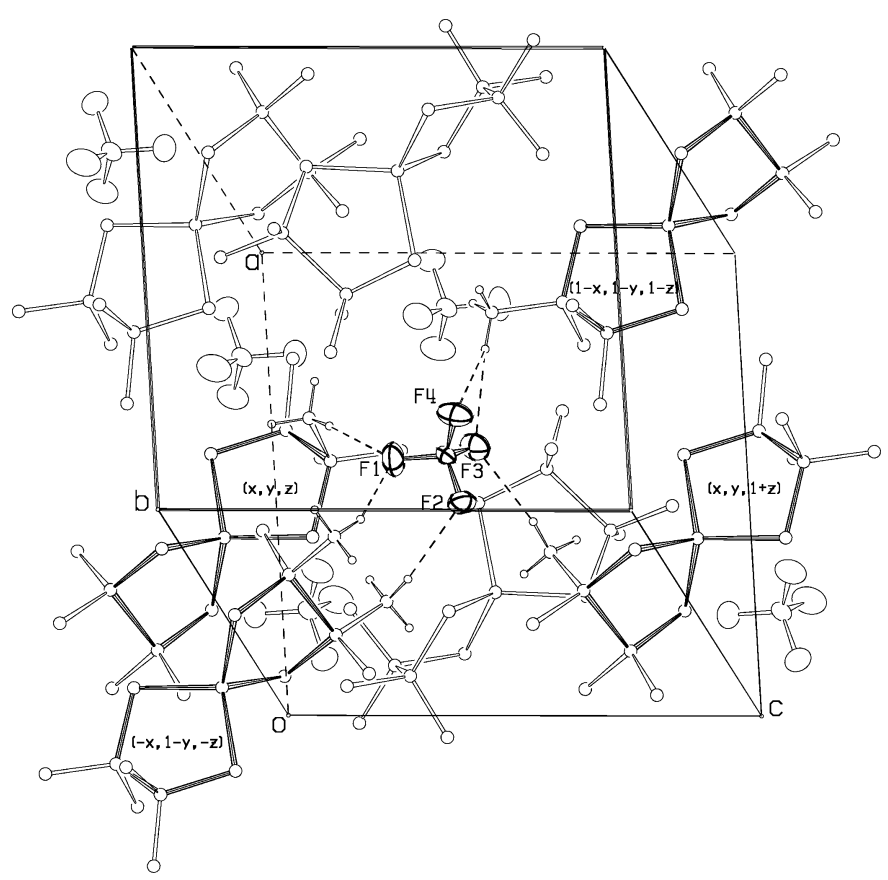

Figure 2

Packing of the title complex, viewed along a direction close to the $b$ axis, with ellipsoid outlines for the anion at $30 \%$ probability. Putative $\mathrm{C}-\mathrm{H} \cdots$ $\mathrm{F}$ hydrogen bonds from four different cations to the $\mathrm{BF}_{4}{ }^{-}$anion are shown. 
containing the diphosphine disulfide ligand of the present study chelated with any metal, $M$, found 11 structures whose coordinates were given. Database $\mathrm{P}-\mathrm{P}$ and $\mathrm{P}=\mathrm{S}$ distances average $2.224(5)$ and $1.993(8) \AA$, while the $M-\mathrm{S}-\mathrm{P}$ and $\mathrm{S}-$ $\mathrm{P}-\mathrm{P}$ angles average $102.1(9)$ and $106.1(6)^{\circ}$, respectively. In the present compound, the $\mathrm{P}=\mathrm{S}$ distances average 1.965 (2) $\AA$ and the average $\mathrm{Cu}-\mathrm{S}-\mathrm{P}$ angle is $98.6(12)^{\circ}$, both close to values for the other copper(I) compound listed, but somewhat less than values for compounds with other metals. The geometry reflects the lack of $\pi$ bonding seen in the copper complexes, as indicated by the small change in $\mathrm{P}=\mathrm{S}$ bond length and $v_{\mathrm{P}-\mathrm{s}}$ vibrational mode upon coordination to copper (Liu et al., 2003). Database torsional angles indicate no preference between $\lambda$ and $\delta$ configurations.

\section{Synthesis and crystallization}

Details of the synthesis and characterization of a number of phosphine sulfides, including the title compound, are given in Meek \& Nicpon (1965).

\section{Refinement details}

Crystal data, data collection and structure refinement details are summarized in Table 3. Each of 18 standard reflections was measured 18-19 times during the $114 \mathrm{~h}$ of data collection. No significant crystal decay was noted; indeed we recorded an overall increase in intensity of $1.6 \%$ over the entire data collection. No corrections were made. Data were collected in two shells, $\theta=0-22.5$ and $\theta=22.5-35^{\circ}$.

The original data reduction deleted reflections with $I<2 \sigma(I)$, and their details are no longer available. Near the end of the final refinements, 2217 missing weak reflections were reinserted into the data file, with $F^{2}$ values set equal to $\sigma\left(F^{2}\right)$ found for reflections with $\mathrm{F}^{2}<3 \sigma\left(F^{2}\right)$, averaged over ten ranges of $\theta$ values. The arbitrary assignment of $F^{2}$ values for these weak reflections perhaps explains the high $K$ value noted for the weakest reflections in the final refinement, where the $F_{\text {cal }}^{2}$ values will be near zero.

The 671 reflection was omitted from the final refinements, due to evidence of a transcription error: the chart record clearly indicates a very weak reflection, while the intensity retrieved from our backup storage is very large. Further, the chart record shows that the very strong 100 reflection was truncated during the scan, and this record was also omitted.

Positions of all non-hydrogen atoms were found by superposition methods. $\mathrm{H}$ atoms in the eight methyl groups were constrained to idealized tetrahedral positions with $\mathrm{C}-\mathrm{H}$ distances of $0.96 \AA$. The methyl torsional angles were refined. The $U_{\text {eq }}$ values for all $\mathrm{H}$ atoms were fixed at 1.2 times the $U_{\text {iso }}$ of their bonded $\mathrm{C}$ atoms.

Initial refinements with anisotropic temperature factors for the heavier atoms and constrained hydrogen atom parameters converged smoothly, to $R_{1}=0.0443$ for 4223 reflections with $F^{2}<2 \sigma$. In case there were systematic anisotropic scaling errors in the data collection that might have affected the detailed electron density around the $\mathrm{BF}_{4}{ }^{-}$anion, the intensity
Table 3

Experimental details.

\begin{tabular}{|c|c|}
\hline \multicolumn{2}{|l|}{ Crystal data } \\
\hline Chemical formula & {$\left[\mathrm{Cu}\left(\mathrm{C}_{4} \mathrm{H}_{12} \mathrm{P}_{2} \mathrm{~S}_{2}\right)_{2}\right] \mathrm{BF}_{4}$} \\
\hline$M_{\mathrm{r}}$ & 522.74 \\
\hline Crystal system, space group & Monoclinic, $P 2_{1} / c$ \\
\hline Temperature (K) & 298 \\
\hline$a, b, c(\AA)$ & $12.388(8), 14.903(10), 12.132(7)$ \\
\hline$\beta\left(^{\circ}\right)$ & $98.02(2)$ \\
\hline$V\left(\AA^{3}\right)$ & $2218(2)$ \\
\hline$Z$ & 4 \\
\hline Radiation type & Mo $K \alpha$ \\
\hline$\mu\left(\mathrm{mm}^{-1}\right)$ & 1.68 \\
\hline Crystal size $(\mathrm{mm})$ & $0.47 \times 0.29 \times 0.25$ \\
\hline \multicolumn{2}{|l|}{ Data collection } \\
\hline Diffractometer & Picker 4-circle \\
\hline Absorption correction & Gaussian (Busing \& Levy, 1957) \\
\hline$T_{\min }, T_{\max }$ & $0.590,0.691$ \\
\hline $\begin{array}{l}\text { No. of measured, independent and } \\
\text { observed }[I>2 \sigma(I)] \text { reflections }\end{array}$ & $6707,6442,4223$ \\
\hline$R_{\text {int }}$ & 0.059 \\
\hline$(\sin \theta / \lambda)_{\max }\left(\AA^{-1}\right)$ & 0.703 \\
\hline \multicolumn{2}{|l|}{ Refinement } \\
\hline$R\left[F^{2}>2 \sigma\left(F^{2}\right)\right], w R\left(F^{2}\right), S$ & $0.040,0.102,1.07$ \\
\hline No. of reflections & 6442 \\
\hline No. of parameters & 207 \\
\hline $\mathrm{H}$-atom treatment & $\mathrm{H}$-atom parameters constrained \\
\hline$\Delta \rho_{\max }, \Delta \rho_{\min }\left(\mathrm{e} \AA^{-3}\right)$ & $0.41,-0.40$ \\
\hline
\end{tabular}

Computer programs: Corfield (1972, 1973), SHELXL97 (Sheldrick, 2008) and ORTEPIII (Burnett \& Johnson, 1996).

data were now smoothed by a 12-parameter model with $X A B S 2$ (Parkin et al., 1995). The smoothing lowered $R_{1}$ to 0.0399 , but had little effect on the electron density or on the atomic parameters: the average $\delta / \sigma$ was 0.9 ; two $\mathrm{F}$ atoms moved by $3 \sigma$.

We made extensive efforts to develop and refine a disordered model for the $\mathrm{BF}_{4}{ }^{-}$anion, in light of the large $U_{i j}$ values for the $\mathrm{F}$ atoms, but were unable to find a model with improved $U_{i j}$ and $R$ values. Difference Fourier syntheses phased on the cation parameters always yielded four large peaks corresponding to the current $\mathrm{F}$ atom positions; final difference Fourier maps did show several much smaller peaks in the vicinity of the $\mathrm{B}$ atom, but no tetrahedral array emerged.

\section{Acknowledgements}

We are grateful for the provision of a crystalline sample by Devon W. Meek, as well as support from the National Science Foundation through equipment grant GP8534 awarded to the Ohio State University, where the experimental work was carried out.

\section{References}

Burnett, M. N. \& Johnson, C. K. (1996). ORTEPIII. Report ORNL6895. Oak Ridge National Laboratory, Tennessee, USA.

Busing, W. R. \& Levy, H. A. (1957). Acta Cryst. 10, 180-182.

Corfield, P. W. R. (1972). Local versions of standard programs, written at Ohio State University.

Corfield, P. W. R., Dabrowiak, J. C. \& Gore, E. S. (1973). Inorg. Chem. 12, 1734-1740. 
Corfield, P. W. R., Paccagnini, M. L. \& Balija, A. M. (2014). Acta Cryst. E70, o400-0401.

Cotton, F. A., Frenz, B. A., Hunter, D. L. \& Mester, Z. C. (1974a). Inorg. Chim. Acta, 11, 111-117.

Cotton, F. A., Frenz, B. A., Hunter, D. L. \& Mester, Z. C. (1974b). Inorg. Chim. Acta, 11, 118-122.

Groom, C. R. \& Allen, F. H. (2014). Angew. Chem. Int. Ed. 53, 662671.
Lee, J. D. \& Goodacre, G. W. (1971). Acta Cryst. B27, 302-307.

Liu, H., Calhorda, M. J., Drew, M. G. B. \& Félix, V. (2003). Inorg. Chim. Acta, 347, 175-180.

Meek, D. W. \& Nicpon, P. (1965). J. Am. Chem. Soc. 87, 4951-4952.

Parkin, S., Moezzi, B. \& Hope, H. (1995). J. Appl. Cryst. 28, 53-56.

Salas, C. O., Tapia, R. A. \& Prieto, Y. (2011). Acta Cryst. E67, o318.

Sheldrick, G. M. (2008). Acta Cryst. A64, 112-122. 


\section{supporting information}

Acta Cryst. (2015). E71, 716-719 [doi:10.1107/S2056989015009913]

\section{Crystal structure of bis(1,1,2,2-tetramethyldiphosphane-1,2-dithione- $\left.\kappa^{2} S, S^{\prime}\right) \operatorname{copper(I)~tetrafluoridoborate}$}

\section{Peter W. R. Corfield and Uwe Seeler}

\section{Computing details}

Data collection: Corfield (1972); cell refinement: Corfield (1972); data reduction: data reduction followed procedures in Corfield et al. (1973) with $p=0.05$, with programs written by Corfield and by Graeme Gainsford; program(s) used to solve structure: local superposition program (Corfield, 1972); program(s) used to refine structure: SHELXL97 (Sheldrick, 2008); molecular graphics: ORTEPIII (Burnett \& Johnson, 1996); software used to prepare material for publication:

SHELXL97 (Sheldrick, 2008).

\section{Bis(1,1,2,2-tetramethyldiphosphane-1,2-dithione- $\left.\kappa^{2} S, S^{\prime}\right) \operatorname{copper}(\mathrm{I})$ tetrafluoridoborate}

Crystal data

$\left[\mathrm{Cu}\left(\mathrm{C}_{4} \mathrm{H}_{12} \mathrm{P}_{2} \mathrm{~S}_{2}\right)_{2}\right] \mathrm{BF}_{4}$

$M_{r}=522.74$

Monoclinic, $P 2_{1} / c$

Hall symbol: -P 2ybc

$a=12.388(8) \AA$

$b=14.903(10) \AA$

$c=12.132(7) \AA$

$\beta=98.02(2)^{\circ}$

$V=2218(2) \AA^{3}$

$Z=4$

\section{Data collection}

Picker 4-circle diffractometer

Radiation source: sealed X-ray tube

Oriented graphite 200 reflection monochromator

$\theta / 2 \theta$ scans

Absorption correction: gaussian (Busing \& Levy, 1957)

$T_{\min }=0.590, T_{\max }=0.691$

6707 measured reflections

\section{Refinement}

Refinement on $F^{2}$ Least-squares matrix: full $R\left[F^{2}>2 \sigma\left(F^{2}\right)\right]=0.040$

$w R\left(F^{2}\right)=0.102$

$S=1.07$

6442 reflections
$F(000)=1064$

$D_{\mathrm{x}}=1.566 \mathrm{Mg} \mathrm{m}^{-3}$

Mo $K \alpha$ radiation, $\lambda=0.71070 \AA$

Cell parameters from 12 reflections

$\theta=2.2-29.4^{\circ}$

$\mu=1.68 \mathrm{~mm}^{-1}$

$T=298 \mathrm{~K}$

Rod, white

$0.47 \times 0.29 \times 0.25 \mathrm{~mm}$

6442 independent reflections 4223 reflections with $I>2 \sigma(I)$

$R_{\text {int }}=0.059$

$\theta_{\max }=30.0^{\circ}, \theta_{\min }=2.2^{\circ}$

$h=-17 \rightarrow 17$

$k=0 \rightarrow 20$

$l=0 \rightarrow 16$

18 standard reflections every 400 reflections intensity decay: -1.6 (1)

\section{7 parameters}

0 restraints

Primary atom site location: heavy-atom method Secondary atom site location: real-space vector search 
Hydrogen site location: inferred from neighbouring sites

H-atom parameters constrained

$$
\begin{aligned}
& w=1 /\left[\sigma^{2}\left(F_{\mathrm{o}}^{2}\right)+(0 . P)^{2}\right] \\
& \text { where } P=\left(F_{\mathrm{o}}^{2}+2 F_{\mathrm{c}}^{2}\right) / 3 \\
& (\Delta / \sigma)_{\max }=0.002 \\
& \Delta \rho_{\max }=0.41 \mathrm{e}^{-3} \\
& \Delta \rho_{\min }=-0.40 \mathrm{e}^{-3}
\end{aligned}
$$

Special details

Geometry. All e.s.d.'s (except the e.s.d. in the dihedral angle between two 1.s. planes) are estimated using the full covariance matrix. The cell e.s.d.'s are taken into account individually in the estimation of e.s.d.'s in distances, angles and torsion angles; correlations between e.s.d.'s in cell parameters are only used when they are defined by crystal symmetry. An approximate (isotropic) treatment of cell e.s.d.'s is used for estimating e.s.d.'s involving 1.s. planes.

Refinement. Refinement of $F^{2}$ against ALL reflections. The weighted $R$-factor $w R$ and goodness of fit $S$ are based on $F^{2}$,

\begin{tabular}{|c|c|c|c|c|}
\hline & $x$ & $y$ & $z$ & $U_{\text {iso }} * / U_{\text {eq }}$ \\
\hline $\mathrm{Cu}$ & 0.21424 & $0.38207(3)$ & -0.01810 & $0.05282(11)$ \\
\hline $\mathrm{S} 1$ & $0.21067(6)$ & $0.39393(6)$ & $0.17147(6)$ & $0.05262(19)$ \\
\hline $\mathrm{P} 2$ & $0.36466(6)$ & $0.41755(5)$ & $0.22442(5)$ & $0.04068(16)$ \\
\hline P3 & $0.46401(5)$ & $0.34297(5)$ & $0.11535(6)$ & $0.04064(16)$ \\
\hline $\mathrm{S} 4$ & $0.40133(6)$ & $0.36787(5)$ & $-0.03970(6)$ & $0.05076(18)$ \\
\hline S5 & $0.11541(7)$ & $0.25250(5)$ & $-0.08606(6)$ & 0.05343 (19) \\
\hline P6 & $0.00172(6)$ & $0.31175(4)$ & $-0.19062(5)$ & $0.04068(16)$ \\
\hline P7 & $0.07818(6)$ & $0.43287(4)$ & $-0.25232(5)$ & $0.03898(15)$ \\
\hline S8 & $0.13575(7)$ & 0.50403 & $-0.12096(6)$ & $0.04904(17)$ \\
\hline $\mathrm{C} 1$ & $0.4022(3)$ & $0.3827(2)$ & $0.3663(2)$ & $0.0736(10)$ \\
\hline $\mathrm{H} 1 \mathrm{~A}$ & 0.3808 & 0.3214 & 0.3743 & $0.088^{*}$ \\
\hline H1B & 0.3661 & 0.4202 & 0.4143 & $0.088^{*}$ \\
\hline $\mathrm{H} 1 \mathrm{C}$ & 0.4797 & 0.3880 & 0.3862 & $0.088 *$ \\
\hline $\mathrm{C} 2$ & 0.4065 & $0.5312(2)$ & 0.2129 & $0.0811(12)$ \\
\hline $\mathrm{H} 2 \mathrm{~A}$ & 0.3843 & 0.5520 & 0.1383 & $0.097 *$ \\
\hline $\mathrm{H} 2 \mathrm{~B}$ & 0.4843 & 0.5350 & 0.2301 & $0.097 *$ \\
\hline $\mathrm{H} 2 \mathrm{C}$ & 0.3733 & 0.5678 & 0.2640 & $0.097 *$ \\
\hline C3 & $0.6038(2)$ & $0.3765(2)$ & $0.1469(3)$ & $0.0691(9)$ \\
\hline $\mathrm{H} 3 \mathrm{~A}$ & 0.6103 & 0.4392 & 0.1310 & $0.083 *$ \\
\hline H3B & 0.6473 & 0.3424 & 0.1024 & $0.083 *$ \\
\hline $\mathrm{H} 3 \mathrm{C}$ & 0.6288 & 0.3658 & 0.2243 & $0.083 *$ \\
\hline $\mathrm{C} 4$ & $0.4556(3)$ & $0.2282(2)$ & $0.1562(3)$ & $0.0713(10)$ \\
\hline $\mathrm{H} 4 \mathrm{~A}$ & 0.3818 & 0.2076 & 0.1382 & $0.086^{*}$ \\
\hline H4B & 0.4778 & 0.2230 & 0.2350 & $0.086^{*}$ \\
\hline $\mathrm{H} 4 \mathrm{C}$ & 0.5026 & 0.1924 & 0.1175 & $0.086^{*}$ \\
\hline $\mathrm{C} 5$ & $-0.0506(3)$ & $0.2437(2)$ & $-0.3084(3)$ & $0.0663(9)$ \\
\hline $\mathrm{H} 5 \mathrm{~A}$ & 0.0082 & 0.2259 & -0.3474 & $0.080^{*}$ \\
\hline H5B & -0.1030 & 0.2775 & -0.3574 & $0.080 *$ \\
\hline $\mathrm{H} 5 \mathrm{C}$ & -0.0850 & 0.1912 & -0.2832 & $0.080 *$ \\
\hline C6 & -0.1121 & $0.3536(2)$ & -0.1301 & $0.0658(9)$ \\
\hline
\end{tabular}
conventional $R$-factors $R$ are based on $F$, with $F$ set to zero for negative $F^{2}$. The threshold expression of $F^{2}>\sigma\left(F^{2}\right)$ is used only for calculating $R$-factors(gt) $e t c$. and is not relevant to the choice of reflections for refinement. $R$-factors based on $F^{2}$ are statistically about twice as large as those based on $F$, and $R$-factors based on ALL data will be even larger.

Fractional atomic coordinates and isotropic or equivalent isotropic displacement parameters $\left(\AA^{2}\right)$ 


$\begin{array}{lllll}\text { H6A } & -0.0866 & 0.3932 & -0.0696 & 0.079^{*} \\ \text { H6B } & -0.1508 & 0.3043 & -0.1029 & 0.079^{*} \\ \text { H6C } & -0.1601 & 0.3858 & -0.1854 & 0.079^{*} \\ \text { C7 } & -0.0204(3) & 0.4916(2) & -0.3488(2) & 0.0595(8) \\ \text { H7A } & -0.0773 & 0.5143 & -0.3104 & 0.071^{*} \\ \text { H7B } & -0.0510 & 0.4513 & -0.4066 & 0.071^{*} \\ \text { H7C } & 0.0145 & 0.5407 & -0.3811 & 0.071^{*} \\ \text { C8 } & 0.1809(3) & 0.3893(2) & -0.3287(3) & 0.0662(9) \\ \text { H8A } & 0.2259 & 0.3475 & -0.2830 & 0.079^{*} \\ \text { H8B } & 0.2250 & 0.4378 & -0.3493 & 0.079^{*} \\ \text { H8C } & 0.1465 & 0.3595 & -0.3945 & 0.079^{*} \\ \text { B } & 0.2831(3) & 0.6284(3) & 0.5224(3) & 0.0677(11) \\ \text { F1 } & 0.2751(3) & 0.6272(2) & 0.4105(2) & 0.1503(14) \\ \text { F2 } & 0.1801(2) & 0.63147(17) & 0.5507(2) & 0.1085(8) \\ \text { F3 } & 0.3356(2) & 0.55271(18) & 0.5658(2) & 0.1177(9) \\ \text { F4 } & 0.3394(2) & 0.70110(19) & 0.5644(3) & 0.1344(11)\end{array}$

Atomic displacement parameters $\left(\AA^{2}\right)$

\begin{tabular}{lllllll}
\hline & $U^{11}$ & $U^{22}$ & $U^{33}$ & $U^{12}$ & $U^{13}$ & $U^{23}$ \\
\hline $\mathrm{Cu}$ & $0.0528(2)$ & $0.0585(2)$ & $0.04281(19)$ & $0.00125(17)$ & $-0.00857(15)$ & $0.00006(15)$ \\
$\mathrm{S} 1$ & $0.0376(3)$ & $0.0772(5)$ & $0.0429(4)$ & $-0.0045(3)$ & $0.0051(3)$ & $-0.0063(3)$ \\
$\mathrm{P} 2$ & $0.0381(3)$ & $0.0449(4)$ & $0.0380(3)$ & $-0.0033(3)$ & $0.0018(3)$ & $-0.0021(3)$ \\
$\mathrm{P} 3$ & $0.0363(3)$ & $0.0429(4)$ & $0.0419(3)$ & $0.0014(3)$ & $0.0024(3)$ & $0.0030(3)$ \\
$\mathrm{S} 4$ & $0.0493(4)$ & $0.0647(5)$ & $0.0384(3)$ & $0.0044(3)$ & $0.0064(3)$ & $0.0030(3)$ \\
$\mathrm{S} 5$ & $0.0608(5)$ & $0.0409(4)$ & $0.0530(4)$ & $0.0009(3)$ & $-0.0116(3)$ & $0.0046(3)$ \\
$\mathrm{P} 6$ & $0.0428(4)$ & $0.0377(3)$ & $0.0396(3)$ & $0.0002(3)$ & $-0.0010(3)$ & $-0.0031(3)$ \\
$\mathrm{P} 7$ & $0.0424(4)$ & $0.0406(3)$ & $0.0334(3)$ & $0.0015(3)$ & $0.0031(3)$ & $-0.0025(3)$ \\
$\mathrm{S} 8$ & $0.0629(4)$ & $0.0370(3)$ & $0.0429(3)$ & $0.0003(3)$ & $-0.0076(3)$ & $-0.0045(3)$ \\
$\mathrm{C} 1$ & $0.069(2)$ & $0.110(3)$ & $0.0381(15)$ & $0.008(2)$ & $-0.0074(15)$ & $0.0023(17)$ \\
$\mathrm{C} 2$ & $0.080(3)$ & $0.0507(19)$ & $0.121(3)$ & $-0.0200(18)$ & $0.045(2)$ & $-0.022(2)$ \\
$\mathrm{C} 3$ & $0.0392(16)$ & $0.096(3)$ & $0.071(2)$ & $-0.0017(17)$ & $0.0006(15)$ & $-0.0036(19)$ \\
$\mathrm{C} 4$ & $0.095(3)$ & $0.0497(18)$ & $0.072(2)$ & $0.0151(18)$ & $0.023(2)$ & $0.0154(16)$ \\
$\mathrm{C} 5$ & $0.074(2)$ & $0.0553(18)$ & $0.0617(19)$ & $-0.0028(16)$ & $-0.0176(17)$ & $-0.0142(15)$ \\
$\mathrm{C} 6$ & $0.060(2)$ & $0.063(2)$ & $0.078(2)$ & $0.0063(16)$ & $0.0247(17)$ & $0.0072(17)$ \\
$\mathrm{C} 7$ & $0.067(2)$ & $0.067(2)$ & $0.0403(14)$ & $0.0046(16)$ & $-0.0050(13)$ & $0.0113(14)$ \\
$\mathrm{C} 8$ & $0.067(2)$ & $0.069(2)$ & $0.067(2)$ & $0.0032(17)$ & $0.0263(18)$ & $-0.0135(16)$ \\
$\mathrm{B}$ & $0.052(2)$ & $0.085(3)$ & $0.062(2)$ & $0.000(2)$ & $-0.0063(18)$ & $0.000(2)$ \\
$\mathrm{F} 1$ & $0.134(3)$ & $0.248(4)$ & $0.0717(17)$ & $0.049(2)$ & $0.0223(17)$ & $0.0095(19)$ \\
$\mathrm{F} 2$ & $0.0738(15)$ & $0.140(2)$ & $0.116(2)$ & $-0.0084(15)$ & $0.0288(14)$ & $-0.0204(16)$ \\
$\mathrm{F} 3$ & $0.102(2)$ & $0.0990(19)$ & $0.145(2)$ & $0.0125(16)$ & $-0.0075(17)$ & $0.0219(18)$ \\
$\mathrm{F} 4$ & $0.103(2)$ & $0.099(2)$ & $0.192(3)$ & $-0.0228(17)$ & $-0.010(2)$ & $-0.009(2)$ \\
& & & & & & \\
\hline & & & & & & \\
\hline
\end{tabular}

Geometric parameters $\left(\AA,{ }^{\circ}\right)$

\begin{tabular}{llll}
\hline $\mathrm{Cu}-\mathrm{S} 1$ & $2.3133(15)$ & $\mathrm{C} 2-\mathrm{H} 2 \mathrm{C}$ & 0.9600 \\
$\mathrm{Cu}-\mathrm{S} 5$ & $2.3719(14)$ & $\mathrm{C} 3-\mathrm{H} 3 \mathrm{~A}$ & 0.9600 \\
$\mathrm{Cu}-\mathrm{S} 4$ & $2.3780(17)$ & $\mathrm{C} 3-\mathrm{H} 3 \mathrm{~B}$ & 0.9600
\end{tabular}




\begin{tabular}{|c|c|c|c|}
\hline $\mathrm{Cu}-\mathrm{S} 8$ & $2.3383(13)$ & $\mathrm{C} 3-\mathrm{H} 3 \mathrm{C}$ & 0.9600 \\
\hline $\mathrm{S} 1-\mathrm{P} 2$ & $1.9580(15)$ & $\mathrm{C} 4-\mathrm{H} 4 \mathrm{~A}$ & 0.9600 \\
\hline $\mathrm{P} 2-\mathrm{C} 2$ & $1.782(3)$ & $\mathrm{C} 4-\mathrm{H} 4 \mathrm{~B}$ & 0.9600 \\
\hline $\mathrm{P} 2-\mathrm{C} 1$ & $1.796(3)$ & $\mathrm{C} 4-\mathrm{H} 4 \mathrm{C}$ & 0.9600 \\
\hline $\mathrm{P} 2-\mathrm{P} 3$ & $2.2262(13)$ & $\mathrm{C} 5-\mathrm{H} 5 \mathrm{~A}$ & 0.9600 \\
\hline $\mathrm{P} 3-\mathrm{C} 4$ & $1.788(3)$ & $\mathrm{C} 5-\mathrm{H} 5 \mathrm{~B}$ & 0.9600 \\
\hline $\mathrm{P} 3-\mathrm{C} 3$ & $1.792(3)$ & $\mathrm{C} 5-\mathrm{H} 5 \mathrm{C}$ & 0.9600 \\
\hline $\mathrm{P} 3-\mathrm{S} 4$ & $1.9677(14)$ & C6-H6A & 0.9600 \\
\hline S5-P6 & $1.9683(13)$ & C6-H6B & 0.9600 \\
\hline $\mathrm{P} 6-\mathrm{C} 6$ & $1.791(3)$ & $\mathrm{C} 6-\mathrm{H} 6 \mathrm{C}$ & 0.9600 \\
\hline $\mathrm{P} 6-\mathrm{C} 5$ & $1.798(3)$ & $\mathrm{C} 7-\mathrm{H} 7 \mathrm{~A}$ & 0.9600 \\
\hline $\mathrm{P} 6-\mathrm{P} 7$ & $2.2166(14)$ & $\mathrm{C} 7-\mathrm{H} 7 \mathrm{~B}$ & 0.9600 \\
\hline $\mathrm{P} 7-\mathrm{C} 8$ & $1.796(3)$ & $\mathrm{C} 7-\mathrm{H} 7 \mathrm{C}$ & 0.9600 \\
\hline $\mathrm{P} 7-\mathrm{C} 7$ & $1.797(3)$ & $\mathrm{C} 8-\mathrm{H} 8 \mathrm{~A}$ & 0.9600 \\
\hline $\mathrm{P} 7-\mathrm{S} 8$ & $1.9637(12)$ & $\mathrm{C} 8-\mathrm{H} 8 \mathrm{~B}$ & 0.9600 \\
\hline $\mathrm{C} 1-\mathrm{H} 1 \mathrm{~A}$ & 0.9600 & $\mathrm{C} 8-\mathrm{H} 8 \mathrm{C}$ & 0.9600 \\
\hline $\mathrm{C} 1-\mathrm{H} 1 \mathrm{~B}$ & 0.9600 & $\mathrm{~B}-\mathrm{F} 4$ & $1.349(5)$ \\
\hline $\mathrm{C} 1-\mathrm{H} 1 \mathrm{C}$ & 0.9600 & $\mathrm{~B}-\mathrm{F} 2$ & $1.369(5)$ \\
\hline $\mathrm{C} 2-\mathrm{H} 2 \mathrm{~A}$ & 0.9600 & $\mathrm{~B}-\mathrm{F} 3$ & $1.370(5)$ \\
\hline $\mathrm{C} 2-\mathrm{H} 2 \mathrm{~B}$ & 0.9600 & $\mathrm{~B}-\mathrm{F} 1$ & $1.347(5)$ \\
\hline $\mathrm{S} 1-\mathrm{Cu}-\mathrm{S} 4$ & $105.69(3)$ & $\mathrm{H} 2 \mathrm{~A}-\mathrm{C} 2-\mathrm{H} 2 \mathrm{C}$ & 109.5 \\
\hline $\mathrm{S} 5-\mathrm{Cu}-\mathrm{S} 8$ & $106.94(5)$ & $\mathrm{H} 2 \mathrm{~B}-\mathrm{C} 2-\mathrm{H} 2 \mathrm{C}$ & 109.5 \\
\hline $\mathrm{S} 1-\mathrm{Cu}-\mathrm{S} 8$ & $114.02(4)$ & $\mathrm{P} 3-\mathrm{C} 3-\mathrm{H} 3 \mathrm{~A}$ & 109.5 \\
\hline $\mathrm{S} 1-\mathrm{Cu}-\mathrm{S} 5$ & $109.10(3)$ & $\mathrm{P} 3-\mathrm{C} 3-\mathrm{H} 3 \mathrm{~B}$ & 109.5 \\
\hline $\mathrm{S} 4-\mathrm{Cu}-\mathrm{S} 5$ & $110.67(4)$ & $\mathrm{H} 3 \mathrm{~A}-\mathrm{C} 3-\mathrm{H} 3 \mathrm{~B}$ & 109.5 \\
\hline $\mathrm{S} 4-\mathrm{Cu}-\mathrm{S} 8$ & $110.46(4)$ & $\mathrm{P} 3-\mathrm{C} 3-\mathrm{H} 3 \mathrm{C}$ & 109.5 \\
\hline $\mathrm{Cu}-\mathrm{S} 1-\mathrm{P} 2$ & $100.78(4)$ & $\mathrm{H} 3 \mathrm{~A}-\mathrm{C} 3-\mathrm{H} 3 \mathrm{C}$ & 109.5 \\
\hline $\mathrm{C} 1-\mathrm{P} 2-\mathrm{C} 2$ & $108.11(18)$ & $\mathrm{H} 3 \mathrm{~B}-\mathrm{C} 3-\mathrm{H} 3 \mathrm{C}$ & 109.5 \\
\hline $\mathrm{C} 1-\mathrm{P} 2-\mathrm{S} 1$ & $111.87(13)$ & $\mathrm{P} 3-\mathrm{C} 4-\mathrm{H} 4 \mathrm{~A}$ & 109.5 \\
\hline $\mathrm{C} 2-\mathrm{P} 2-\mathrm{S} 1$ & $115.18(14)$ & $\mathrm{P} 3-\mathrm{C} 4-\mathrm{H} 4 \mathrm{~B}$ & 109.5 \\
\hline $\mathrm{C} 1-\mathrm{P} 2-\mathrm{P} 3$ & $109.56(13)$ & $\mathrm{H} 4 \mathrm{~A}-\mathrm{C} 4-\mathrm{H} 4 \mathrm{~B}$ & 109.5 \\
\hline $\mathrm{C} 2-\mathrm{P} 2-\mathrm{P} 3$ & $103.71(12)$ & $\mathrm{P} 3-\mathrm{C} 4-\mathrm{H} 4 \mathrm{C}$ & 109.5 \\
\hline $\mathrm{S} 1-\mathrm{P} 2-\mathrm{P} 3$ & $108.02(5)$ & $\mathrm{H} 4 \mathrm{~A}-\mathrm{C} 4-\mathrm{H} 4 \mathrm{C}$ & 109.5 \\
\hline $\mathrm{C} 4-\mathrm{P} 3-\mathrm{C} 3$ & $107.42(17)$ & $\mathrm{H} 4 \mathrm{~B}-\mathrm{C} 4-\mathrm{H} 4 \mathrm{C}$ & 109.5 \\
\hline $\mathrm{C} 4-\mathrm{P} 3-\mathrm{S} 4$ & $114.52(12)$ & $\mathrm{P} 6-\mathrm{C} 5-\mathrm{H} 5 \mathrm{~A}$ & 109.5 \\
\hline $\mathrm{C} 3-\mathrm{P} 3-\mathrm{S} 4$ & $113.12(12)$ & $\mathrm{P} 6-\mathrm{C} 5-\mathrm{H} 5 \mathrm{~B}$ & 109.5 \\
\hline $\mathrm{C} 4-\mathrm{P} 3-\mathrm{P} 2$ & $104.74(12)$ & $\mathrm{H} 5 \mathrm{~A}-\mathrm{C} 5-\mathrm{H} 5 \mathrm{~B}$ & 109.5 \\
\hline $\mathrm{C} 3-\mathrm{P} 3-\mathrm{P} 2$ & $109.37(12)$ & $\mathrm{P} 6-\mathrm{C} 5-\mathrm{H} 5 \mathrm{C}$ & 109.5 \\
\hline $\mathrm{S} 4-\mathrm{P} 3-\mathrm{P} 2$ & $107.27(5)$ & $\mathrm{H} 5 \mathrm{~A}-\mathrm{C} 5-\mathrm{H} 5 \mathrm{C}$ & 109.5 \\
\hline $\mathrm{P} 3-\mathrm{S} 4-\mathrm{Cu}$ & $99.91(5)$ & $\mathrm{H} 5 \mathrm{~B}-\mathrm{C} 5-\mathrm{H} 5 \mathrm{C}$ & 109.5 \\
\hline $\mathrm{Cu}-\mathrm{S} 5-\mathrm{P} 6$ & $98.45(5)$ & $\mathrm{P} 6-\mathrm{C} 6-\mathrm{H} 6 \mathrm{~A}$ & 109.5 \\
\hline $\mathrm{C} 5-\mathrm{P} 6-\mathrm{C} 6$ & $107.78(17)$ & $\mathrm{P} 6-\mathrm{C} 6-\mathrm{H} 6 \mathrm{~B}$ & 109.5 \\
\hline $\mathrm{C} 5-\mathrm{P} 6-\mathrm{S} 5$ & $113.90(12)$ & $\mathrm{H} 6 \mathrm{~A}-\mathrm{C} 6-\mathrm{H} 6 \mathrm{~B}$ & 109.5 \\
\hline $\mathrm{C} 6-\mathrm{P} 6-\mathrm{S} 5$ & $115.20(13)$ & $\mathrm{P} 6-\mathrm{C} 6-\mathrm{H} 6 \mathrm{C}$ & 109.5 \\
\hline $\mathrm{C} 5-\mathrm{P} 6-\mathrm{P} 7$ & $108.35(12)$ & $\mathrm{H} 6 \mathrm{~A}-\mathrm{C} 6-\mathrm{H} 6 \mathrm{C}$ & 109.5 \\
\hline $\mathrm{C} 6-\mathrm{P} 6-\mathrm{P} 7$ & $104.64(12)$ & $\mathrm{H} 6 \mathrm{~B}-\mathrm{C} 6-\mathrm{H} 6 \mathrm{C}$ & 109.5 \\
\hline $\mathrm{S} 5-\mathrm{P} 6-\mathrm{P} 7$ & $106.38(6)$ & $\mathrm{P} 7-\mathrm{C} 7-\mathrm{H} 7 \mathrm{~A}$ & 109.5 \\
\hline
\end{tabular}




$\begin{array}{llll}\text { C8-P7-C7 } & 107.79(16) & \text { P7-C7-H7B } & 109.5 \\ \text { C8-P7-S8 } & 114.24(13) & \text { H7A-C7-H7B } & 109.5 \\ \text { C7-P7-S8 } & 113.73(11) & \text { P7-C7-H7C } & 109.5 \\ \text { C8-P7-P6 } & 104.29(12) & \text { H7A-C7-H7C } & 109.5 \\ \text { C7-P7-P6 } & 109.48(12) & \text { H7B-C7-H7C } & 109.5 \\ \text { S8-P7-P6 } & 106.83(5) & \text { P7-C8-H8A } & 109.5 \\ \text { P7-S8-Cu } & 95.17(6) & \text { P7-C8-H8B } & 109.5 \\ \text { P2-C1-H1A } & 109.5 & \text { H8A-C8-H8B } & 109.5 \\ \text { P2-C1-H1B } & 109.5 & \text { P7-C8-H8C } & 109.5 \\ \text { H1A-C1-H1B } & 109.5 & \text { H8A-C8-H8C } & 109.5 \\ \text { P2-C1-H1C } & 109.5 & \text { H8B-C8-H8C } & 109.5 \\ \text { H1A-C1-H1C } & 109.5 & \text { F1-B-F2 } & 108.2(3) \\ \text { H1B-C1-H1C } & 109.5 & \text { F1-B-F3 } & 109.9(4) \\ \text { P2-C2-H2A } & 109.5 & \text { F1-B-F4 } & 110.6(4) \\ \text { P2-C2-H2B } & 109.5 & \text { F2-B-F3 } & 109.9(4) \\ \text { H2A-C2-H2B } & 109.5 & \text { F2-B-F4 } & 109.2(4) \\ \text { P2-C2-H2C } & 109.5 & \text { F3-B-F4 } & 108.9(3) \\ \text { Cu-S1-P2-P3 } & & \text { Cu-S5-P6-P7 } & \\ \text { S1-P2-P3-S4 } & -33.85(5) & \text { S5-P6-P7-S8 } & 31.27(5) \\ \text { P2-P3-S4-Cu } & 47.97(6) & \text { P6-P7-S8-Cu } & -56.37(6)\end{array}$

Hydrogen-bond geometry $\left(\AA,{ }^{\circ}\right)$

\begin{tabular}{lllll}
\hline$D-\mathrm{H} \cdots A$ & $D-\mathrm{H}$ & $\mathrm{H} \cdots A$ & $D \cdots A$ & $D-\mathrm{H} \cdots A$ \\
\hline $\mathrm{C} 1-\mathrm{H} 1 C \cdots \mathrm{F} 4^{\mathrm{i}}$ & 0.96 & 2.60 & $3.430(5)$ & 145 \\
$\mathrm{C} 1-\mathrm{H} 1 C \cdots \mathrm{F} 3^{\mathrm{i}}$ & 0.96 & 2.45 & $3.378(5)$ & 164 \\
$\mathrm{C} 2-\mathrm{H} 2 C \cdots \mathrm{F} 1$ & 0.96 & 2.46 & $3.397(5)$ & 167 \\
$\mathrm{C} 5-\mathrm{H} 5 B \cdots \mathrm{F} 1^{\mathrm{ii}}$ & 0.96 & 2.57 & $3.465(5)$ & 156 \\
$\mathrm{C} 7-\mathrm{H} 7 B^{\cdots} \cdots \mathrm{F} 2^{\mathrm{ii}}$ & 0.96 & 2.52 & $3.453(4)$ & 164 \\
$\mathrm{C} 8-\mathrm{H} 8 B \cdots \mathrm{F} 3^{\mathrm{iii}}$ & 0.96 & 2.50 & $3.454(5)$ & 171 \\
\hline
\end{tabular}

Symmetry codes: (i) $-x+1,-y+1,-z+1$; (ii) $-x,-y+1,-z$; (iii) $x, y, z-1$. 